Allison Crain, MD Department of Family,

Community and Preventive Medicine, University of Arizona College of Medicine, Phoenix, and

St. Joseph's Hospital Family Medicine Residency at Creighton University Arizona Health Education Alliance, Phoenix

\section{DAllison.Crain@ dignityhealth.org}

The author reported no potential conflict of interest relevant to this article.

doi: 10.12788/jfp.0127

\title{
Breaking the cycle of medication overuse headache
}

\author{
Care of this disorder can be complex-from ruling out \\ another secondary cause of headache to supervising \\ detox from abortives, providing preventives, and \\ educating often-fearful patients.
}

\section{PRACTICE RECOMMENDATIONS \\ $\mathrm{M}$}

> Avoid prescribing barbiturates or opioids for a headache disorder. A

> Limit use of a headacheabortive medication to twice a week when starting a patient on the drug. (c)

> Consider providing bridging therapy during detoxification of the overused medication. (c)

> Do not provide a preventive medication without withdrawing the overused agent. (A)

Strength of recommendation (SOR)

A Good-quality patient-oriented evidence

B Inconsistent or limited-quality patient-oriented evidence

C Consensus, usual practice, opinion, disease-oriented evidence, case series edication overuse headache ( $\mathrm{MOH})$, a secondary headache diagnosis, is a prevalent phenomenon that complicates headache diagnosis and treatment, increases the cost of care, and reduces quality of life. Effective abortive medication is essential for the headache sufferer; when an abortive is used too frequently, however, headache frequency increases-potentially beginning a cycle in which the patient then takes more medication to abort the headache. Over time, the patient suffers from an ever-increasing number of headaches, takes even more abortive medication, and so on. In the presence of $\mathrm{MOH}$, there is a reduction in pain response to preventive and abortive treatments; when medication overuse is eliminated, pain response improves. ${ }^{1}$

Although MOH is well recognized among headache specialists, the condition is often overlooked in primary care. Since headache is a top complaint in primary care, however, and prevention is a major goal in family medicine, the opportunity for you to recognize, treat, and prevent $\mathrm{MOH}$ is significant. In fact, a randomized controlled trial showed that brief patient education about headache care and $\mathrm{MOH}$ provided by a primary care physician can lead to a significant reduction in headache frequency among patients with $\mathrm{MOH} .{ }^{2}$

This article reviews the recognition and diagnosis of $\mathrm{MOH}$, based on historical features and current criteria; addresses risk factors for abortive medication overuse and how to withdraw an offending agent; and explores the value of bridging and preventive therapies to reduce the overall frequency of headache.

\section{What defines $\mathrm{MOH}$ ?}

Typically, MOH is a chronification of a primary headache disorder. However, in patients with a history of migraine who are undergoing treatment for another chronic pain condition with an opioid or other analgesic, $\mathrm{MOH}$ can be induced. ${ }^{3} \mathrm{An}$ 


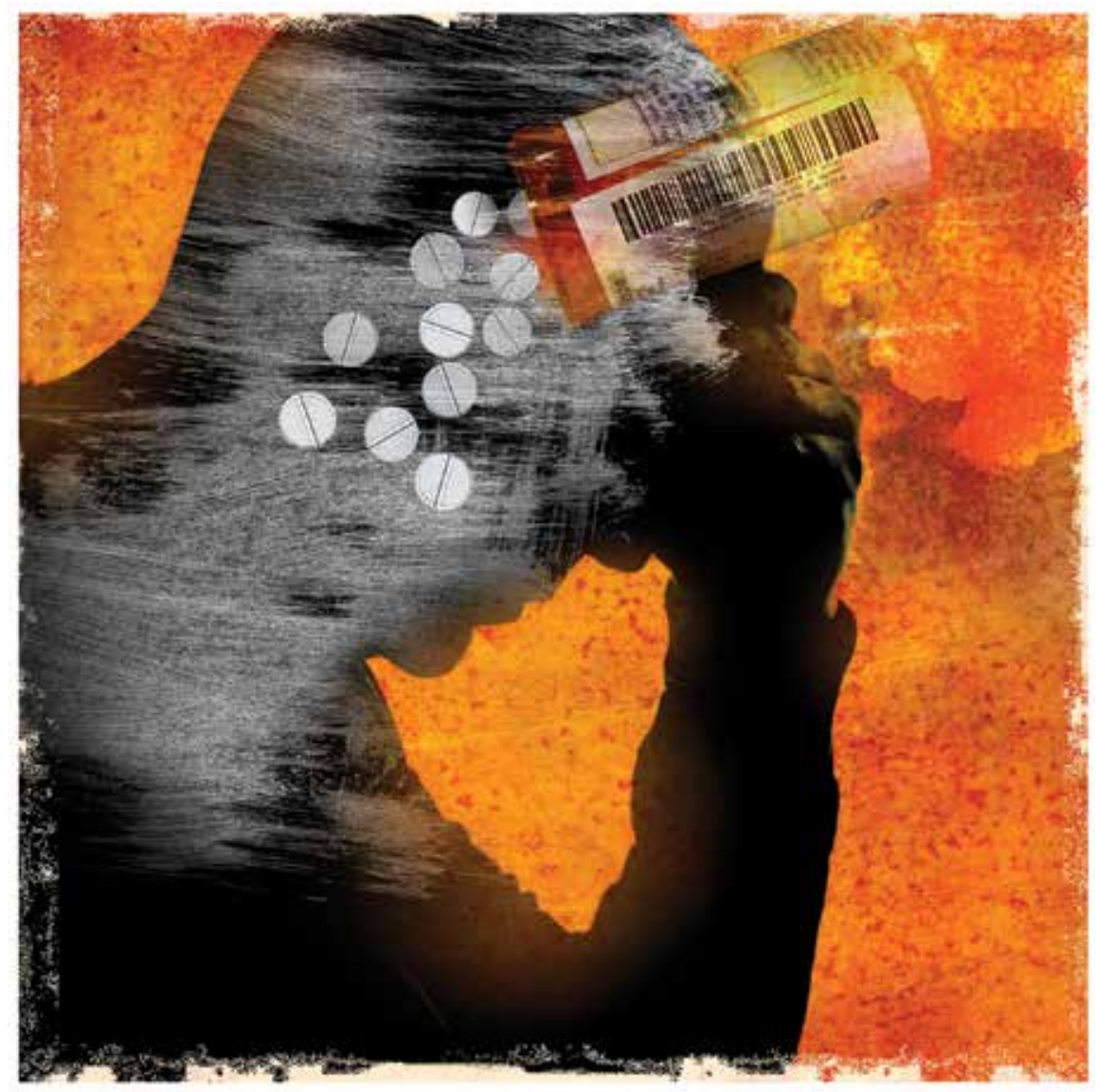

Although medication overuse headache is well recognized among headache specialists, the condition is often overlooked in primary care.

increase in the frequency of headache raises the specter of a concomitant increase in the level of disability ; psychiatric comorbidity ${ }^{5}$; and more headache days, with time lost from school and work.

The Migraine Disability Assessment (MIDAS) questionnaire, a validated instrument that helps the provider (1) measure the impact that headache has on a patient's life and (2) follow treatment progress, also provides information to employers and insurance companies on treatment coverage and the need for work modification. The MIDAS score is 3 times higher in patients with $\mathrm{MOH}$ than in patients with episodic migraine. ${ }^{6,7}$

The annual associated cost per person of MOH has been estimated at \$4000, resulting in billions of dollars in associated costs ${ }^{8}$; most of these costs are related to absenteeism and disability. After detoxification for $\mathrm{MOH}$, annual outpatient medication costs are reduced by approximately $24 \% .^{9}$

\section{Efforts to solve a common problem create another}

Headache affects nearly $50 \%$ of the general population worldwide, ${ }^{10}$ accounting for about $4 \%$ of primary care visits ${ }^{11}$ and approximately
$20 \%$ of outpatient neurology consultations. ${ }^{12}$ Although inpatient stays for headache are approximately half the duration of the overall average hospital stay, headache accounts for 3\% of admissions. ${ }^{13}$ According to the Global Burden of Disease study, tension-type headache, migraine, and $\mathrm{MOH}$ are the 3 most common headache disorders. ${ }^{10}$ Headache is the second leading cause of disability among people 15 to 49 years of age. ${ }^{10}$

The prevalence of $\mathrm{MOH}$ in the general population is $2 \% .^{7,14,15}$ A population-based study showed that the rate of progression from episodic headache $(<15 \mathrm{~d} / \mathrm{mo})$ to chronic headache $(\geq 15 \mathrm{~d} / \mathrm{mo})$ in the general population is $2.5 \%$ per year ${ }^{16}$; however, progression to chronic headache is $14 \%$ per year in patients with medication overuse. Onethird of the general population with chronic migraine overuses symptomatic medication; in US headache clinics, roughly one-half of patients with chronic headache overuse acute medication. ${ }^{6}$

\section{Definitions and diagnosis}

$\mathrm{MOH}$ is a secondary headache diagnosis in the third edition of the International Classification of Headache Disorders (ICHD-3) 


\section{$>$}

The time it takes to develop medication overuse headache is shortest with triptans, followed by ergots, then analgesics.
(TABLE 1), ${ }^{17}$ which lists diagnostic criteria for recognized headache disorders.

I Terminology. $\mathrm{MOH}$ has also been called rebound headache, drug-induced headache, and transformed migraine, but these terms are outdated and are not formal diagnoses. Patients sometimes refer to substance-withdrawal headaches (not discussed in this article) as rebound headaches, so clarity is important when discussing headache with patients: namely, that $\mathrm{MOH}$ is an exacerbation of an existing headache condition caused by overuse of abortive headache medications, including analgesics, combination analgesics, triptans, barbiturates, and opioids.

$\mathrm{MOH}$ was recognized in the early 1950s and fully differentiated as a diagnosis in 2005 in the second edition of the ICHD. The disorder is subcategorized by offending abortive agent (TABLE $2^{17}$ ) because the frequency of analgesic use required to develop $\mathrm{MOH}$ differs by agent.

I Risk factors for $\mathrm{MOH}$ and chronification of a primary headache disorder. There are several risk factors for developing $\mathrm{MOH}$, and others that contribute to increasing headache frequency in general (TABLE $3^{5,14,18-23}$ ). Some risk factors are common to each. All are important to address because some are modifiable.

I Pathophysiology. The pathophysiology and psychology behind MOH are largely unknown. Physiologic changes in pain processing and functional imaging changes have been demonstrated in patients with $\mathrm{MOH}$, both of which are reversible upon withdrawal of medication. ${ }^{23}$ Genetic factors and changes in hormone and neurotransmitter levels are found in $\mathrm{MOH}$ patients; this is not the case in patients who have an episodic headache pattern only. ${ }^{24}$

I Presentation. Diagnostic criteria for MOH do not include clinical characteristics. Typically, the phenotype of $\mathrm{MOH}$ in a given patient is similar to the underlying primary headache ${ }^{25}$-although this principle can be complicated to tease out because these medications can suppress some symptoms. Diagnosis of a primary headache disorder should be documented along with the diagnosis of $\mathrm{MOH}$.
Medication overuse can exist without $\mathrm{MOH}$ : Not every patient who frequently uses an abortive medication develops $\mathrm{MOH}$.

\section{Treatment is multifaceted- and can become complex}

Mainstays of treatment of $\mathrm{MOH}$ are education about the disorder and detoxification from the overused agent, although specific treatments can differ depending on the agent involved, the frequency and duration of its use, and a patient's behavioral patterns and psychiatric comorbidities. Often, a daily medication to prevent headache is considered upon, or after, withdrawal of the offending agent. The timing of introducing a preventive might impact its effectiveness. Some refractory cases require more intensive therapy, including hospitalization at a specialized tertiary center.

But before we look at detoxification from an overused agent, it's important to review one of the best strategies of all in combatting $\mathrm{MOH}$.

\section{First and best strategy: Avoid onset of $\mathrm{MOH}$}

I Select an appropriate abortive to reduce the risk of $\mathrm{MOH}$. With regard to specific acute headache medications, some nuances other than type of headache should be considered. Nonsteroidal anti-inflammatory drugs (NSAIDs) are recommended as abortive therapy by the American Headache Society for their efficacy, favorable adverse effect profile, and low cost. NSAIDs are protective against development of $\mathrm{MOH}$ if a patient's baseline headache frequency is $<10 / \mathrm{mo}$; at a frequency of 10 to $14 \mathrm{~d} / \mathrm{mo}$, however, the risk of $\mathrm{MOH}$ increases when using an NSAID. ${ }^{6}$ A similar effect has been seen with triptans. ${ }^{16}$ Longeracting NSAIDs, such as nabumetone and naproxen, have been proposed as less likely to cause $\mathrm{MOH}$, and are even used as bridging therapy sometimes (as long as neither of these was the overused medication). ${ }^{26}$

The time it takes to develop $\mathrm{MOH}$ is shortest with triptans, followed by ergots, then analgesics. ${ }^{27}$

Prospective cohort studies ${ }^{6,16}$ have shown that barbiturates and opioids are more like- 
ly to induce $\mathrm{MOH}$; for that reason, agents in these analgesic classes are almost universally avoided unless no other medically acceptable options exist. Using barbituratecontaining compounds or opioids $>4 \mathrm{~d} / \mathrm{mo}$ exponentially increases the likelihood of $\mathrm{MOH}$.

Promising preclinical data demonstrate that the gepant, or small-molecule calcitonin gene-related peptide (CGRP) receptor antagonist, class of medications used as abortive therapy does not induce medication overuse cutaneous allodynia. ${ }^{28}$

I Provide education. Primary prevention of MOH involves (1) increasing patients' awareness of how to take medications appropriately and (2) restricting intake of overthe-counter abortive medications. Often, the expert recommendation is to limit abortives to approximately $2 \mathrm{~d} / \mathrm{wk}$ because more frequent use places patients at risk of further increased use and subsequent $\mathrm{MOH}$.

A randomized controlled trial in Norway compared outcomes in 2 groups of patients with $\mathrm{MOH}$ : One group was given advice on the disorder by a physician; the other group was not provided with advice. In the "business-as-usual" group, there was no significant improvement; however, when general practitioners provided simple advice (lasting roughly 9 minutes) about reducing abortive medication use to a safe level and cautioned patients that they would be "feeling worse before feeling better," headache days were reduced by approximately 8 per month and medication days, by 16 per month. ${ }^{2}$

A subsequent, long-term follow-up study $^{29}$ of patients from the Norway trial ${ }^{2}$ who had been given advice and education showed a relapse rate (ie, into overuse of headache medication) of only $8 \%$ and sustained reduction of headache days and medication use at 16 months.

I Offer support and other nondrug interventions. A recent review of 3 studies $^{23}$ recommended that extra support for patients from a headache nurse, close followup, keeping an electronic diary that provides feedback, and undertaking a short course of psychotherapy can reduce medication overuse and prevent relapse.
TABLE 1

\section{Diagnostic criteria for medication overuse headache ${ }^{17}$}

Headache that occurs $\geq 15 \mathrm{~d} / \mathrm{mo}$ in a patient with a pre-existing headache disorder

Regular overuse for $>3$ mo of $\geq 1$ drugs that can be taken for acute or symptomatic (or both) treatment of headache ${ }^{a}$

Headache that is not better accounted for by another diagnosis in The International Classification of Headache Disorders, 3rd edition ${ }^{17}$

a Patients should be coded for 1 or more subtypes of $\mathbf{8 . 2}$ Medication-overuse headache according to the specific medication(s) overused and the criteria for each subtype, as follows:

- A patient who fulfills criteria for 8.2.2 Triptan-overuse headache and criteria for a subtype of 8.2.3 Non-opioid analgesic-overuse headache should receive both codes. Exception: A patient overuses a combination-analgesic medication, which is coded 8.2.5 Combination-analgesic-overuse headache and not according to each constituent of the combination-analgesic medication.

- A patient who uses multiple drugs for acute or symptomatic treatment of headache might do so in a manner that constitutes overuse, even though no individual drug or class of drug is overused; such a patient should be coded 8.2.6 Medication-overuse headache attributed to multiple drug classes not individually overused.

- A patient who is clearly overusing multiple drugs for acute or symptomatic treatment of headache but cannot give an adequate account of the names or quantities of those drugs is coded 8.2.7 Medication-overuse headache attributed to unspecified or unverified overuse of multiple drug classes until better information is available. Almost all such cases necessitate follow-up using a diary.

For more information on the classification of medication overuse headache, see: https://ichd-3.org/8-headache-attributed-to-a-substance-or-its-withdrawal/8-2medication-overuse-headache-moh/. ${ }^{17}$

\section{If MOH develops, initiate withdrawal, introduce a preventive}

I Withdraw overused medication. Most current evidence suggests that withdrawal of the offending agent is the most effective factor in reducing headache days and improving quality of life. A randomized controlled trial compared the effects of (1) complete and immediate withdrawal of an abortive medication with (2) reducing its use (ie, limiting intake to $2 \mathrm{~d} / \mathrm{wk}$ ), on headache frequency, disability, and quality of life. ${ }^{30}$ There was a reduction of headache days in both groups; however, reduction was much greater at 2 months in the complete withdrawal group than in the restricted intake group (respectively, a $41 \%$ and a $26 \%$ reduction in headache days per month). This effect was sustained at 6 and 12 months in both groups. The study confirmed the results of earlier research ${ }^{2,15}$ : Abrupt withdrawal leads to reversion to an episodic pattern at 2 to 6 months in approximately $40 \%$ to $60 \%$ of patients. 
TABLE 2

\section{Medication overuse headache subcategories by offending abortive agent ${ }^{17}$}

\begin{tabular}{l|l}
\hline Subgroup of abortive medication & Extent of abortive medication use \\
\hline - Ergotamine (or ergot alkaloids) & $\geq 10 \mathrm{~d} / \mathrm{mo}$ \\
- Combination analgesics ( $\geq 2$ analgesics or an analgesic \\
combined with caffeine) \\
- Opioids & \\
- Triptans & $\geq 15 \mathrm{~d} / \mathrm{mo}$ \\
\hline Nonopioid analgesic overuse headache & \\
- Acetaminophen & \\
- Acetylsalicylic acid & \\
- Nonsteroidal anti-inflammatory drugs & \\
- Other & \\
\hline
\end{tabular}

More studies are needed to determine the most appropriate treatment course for $\mathrm{MOH}$; however, complete withdrawal of the causative drug is the most important intervention.

I Consider withdrawal plus preventive treatment. Use of sodium valproate, in addition to medication overuse detoxification, led to a significant reduction in headache days and improvement in quality of life at 12 weeks but no difference after 24 weeks, compared with detoxification alone in a randomized, double-blind, placebo-controlled study. ${ }^{31}$

A study of 61 patients showed a larger reduction (by $7.2 \mathrm{~d} / \mathrm{mo}$ ) in headache frequency with any preventive medication in addition to medication withdrawal, compared to withdrawal alone (by $4.1 \mathrm{~d} / \mathrm{mo}$ ) after 3 months; however, the relative benefit was gone at 6 months. ${ }^{32}$

A study of 98 patients compared immediate and delayed initiation of preventive medication upon withdrawal of overused abortive medication. ${ }^{33}$ Response was defined as a $>50 \%$ reduction in headache frequency and was similar in both groups; results showed a $28 \%$ response with immediate initiation of a preventive; a $23 \%$ response with delayed (ie, 2 months after withdrawal) initiation; and a $48 \%$ response in both groups at 12 months.

Collectively, these studies suggest that adding a preventive medication at the time of withdrawal has the potential to reduce headache frequency more quickly than withdrawal alone. However, after 3 to 6 months, the outcome of reduced headache frequency is the same whether or not a preventive medication is used-as long as the offending agent has been withdrawn.

I Do preventives work without withdrawing overused medication? Patients with $\mathrm{MOH}$ often show little or no improvement with addition of a preventive medication only; their response to a preventive improves after withdrawal of the overused medication. Patients without previous headache improvement after addition of a preventive, who also did not improve 2 months after withdrawal, then demonstrated an overall reduction in headache by $26 \%$ when a preventive was reintroduced after withdrawal. ${ }^{2}$

I The research evidence for preventives. Medications for headache prevention have not been extensively evaluated specifically for treating $\mathrm{MOH}$. Here is what's known:

- Flunarizine, amitriptyline, and betablockers usually are ineffective for MOH. ${ }^{24}$

- Results for topiramate are mixed: A small, double-blind, placebocontrolled chronic migraine study in Europe showed that, in a subgroup of patients with $\mathrm{MOH}$, topiramate led to a small but significant reduction (3.5 d/mo) in headache frequency, compared to placebo. ${ }^{27} \mathrm{~A}$ similar study done in the United States did not show a significant difference between the active-treatment and placebo groups. ${ }^{34}$ 
TABLE 3

Risk factors for increasing headache frequency $y^{5,14,18-23}$

\begin{tabular}{|c|c|}
\hline Headache chronification ${ }^{a}$ & Medication overuse headache ${ }^{a}$ \\
\hline Allodynia ${ }^{21}$ & Baseline attack frequency $7-14 \mathrm{~d} / \mathrm{mo}^{19}$ \\
\hline Asthma, chronic bronchitis, sinusitis ${ }^{21}$ & Body mass index $>30^{23}$ \\
\hline Baseline attack frequency $\geq 10-15 \mathrm{~d} / \mathrm{mo}^{20}$ & Chronic noncephalic pain ${ }^{19}$ \\
\hline Chronic noncephalic pain ${ }^{21}$ & Family history of medication overuse headache ${ }^{14}$ \\
\hline Depression, anxiety ${ }^{19,21}$ & Female $14,19,22$ \\
\hline Family history of migraine or substance abuse ${ }^{18}$ & Gastrointestinal complaints ${ }^{18,19}$ \\
\hline Female $^{18,19}$ & Insomnia ${ }^{19}$ \\
\hline Gastrointestinal complaints ${ }^{18,19}$ & Low education ${ }^{18,19}$ \\
\hline Hypertension ${ }^{18}$ & Low socioeconomic status ${ }^{14}$ \\
\hline Low education ${ }^{18,19}$ & Married $^{14}$ \\
\hline Married ${ }^{18}$ & Obsessive-compulsive disorder, anxiety disorder, mood disorder \\
\hline Medication overuse $\mathrm{e}^{21}$ & Physical inactivity ${ }^{19}$ \\
\hline Menopause $^{18}$ & Primary headache is migraine ${ }^{18}$ \\
\hline No migraine remission during pregnancy ${ }^{18}$ & Sedative-hypnotic use ${ }^{18,19}$ \\
\hline Not using an oral contraceptive ${ }^{18}$ & Sick leave $>2 \mathrm{wk}$ in the previous year ${ }^{19}$ \\
\hline Primary headache is nonmigrainous ${ }^{19}$ & Smoking ${ }^{19}$ \\
\hline Snoring, obstructive sleep apnea, insomnia 19,21 & Whiplash injury ${ }^{19}$ \\
\hline Unemployed $^{18}$ & Younger $^{\mathrm{b}}$ age $^{19}$ \\
\hline Whiplash injury ${ }^{19}$ & \\
\hline
\end{tabular}

- Findings regarding onabotulinumtoxinA are intriguing: In a posthoc analysis of onabotulinumtoxinA to treat chronic migraine, patients with $\mathrm{MOH}$ who did not undergo detoxification had an $8 \mathrm{~d} /$ mo greater reduction in headache, compared to placebo. ${ }^{35}$ However, when compared to placebo in conjunction with detoxification, onabotulinumtoxinA demonstrated no benefit. ${ }^{36}$

- Newer CGRP antagonist and CGRP receptor antagonist monoclonal antibodies are successful preventive medications that have demonstrated a reduction in acute medication use days per month and headache days per month $^{37}$; these compounds have not been compared to withdrawal alone.

\section{Reducing the severity} and duration of withdrawal symptoms

Withdrawal from overused abortive head- ache medications can lead to worsening headache, nausea, vomiting, hypotension, tachycardia, sleep disturbances, restlessness, anxiety, and nervousness. Symptoms usually last 2 to 10 days but can persist for as long as 4 weeks; duration of withdrawal symptoms varies with the medication that is being overused. In patients who have used a triptan, for example, mean duration of withdrawal is 4.1 days; ergotamine, 6.7 days; and NSAIDs, 9.5 days. ${ }^{23}$ Tapered withdrawal is sometimes recommended with opioids and barbiturates to reduce withdrawal symptoms. It is unclear whether starting a preventive medication during withdrawal assists in reducing withdrawal symptoms. ${ }^{38}$

I Bridging therapy to reduce symptoms of withdrawal is often provided despite debatable utility. Available evidence does not favor one agent or method but suggests some strategies that could be helpful:

- A prednisone taper has a potential role during the first 6 days of with- 


\section{$>$}

Reduce the risk of medication overuse headache by selecting an appropriate abortive;

NSAIDs are recommended for their efficacy, favorable adverse effect profile, and low cost. drawal by reducing rebound headache and withdrawal symptoms ${ }^{39}$; however, oral prednisolone has been shown to have no benefit. ${ }^{40}$

- Alone, IV methylprednisolone seems not to be of benefit; however, in a retrospective study of 94 patients, IV methylprednisolone plus diazepam for 5 days led to a significant reduction in headache frequency and drug consumption that was sustained after 3 months. ${ }^{41}$

- Celecoxib was compared to prednisone over a 20-day course: a celecoxib dosage of $400 \mathrm{mg} / \mathrm{d}$ for the first 5 days, tapered by $100 \mathrm{mg}$ every 5 days, and an oral prednisone dosage of $75 \mathrm{mg} / \mathrm{d}$ for the first 5 days, then tapered every 5 days. Patients taking celecoxib had lower headache intensity but there was no difference in headache frequency and acute medication intake between the groups. ${ }^{42}$

I Other strategies. Using antiemetics and NSAIDs to reduce withdrawal symptoms is widely practiced, but no placebocontrolled trials have been conducted to support this strategy.

Patients in withdrawal might be more likely to benefit from inpatient care if they have a severe comorbidity, such as opioid or barbiturate use; failure to respond to, tolerate, or adhere to treatment; or relapse after withdrawal. ${ }^{38}$

Cognitive behavioral therapy, exercise, a headache diary, and biofeedback should be considered in every patient's treatment strategy because a multidisciplinary approach increases adherence and leads to improvement in headache frequency and a decrease in disability and medication use. ${ }^{43}$

\section{Predictors of Tx success}

A prospective cohort study determined that the rate of $\mathrm{MOH}$ relapse is $31 \%$ at 6 months, $41 \%$ at 1 year, and $45 \%$ at 4 years, with the highest risk of relapse during the first year. ${ }^{44}$ Looking at the correlation between type of medication overused and relapse rate, the research indicates that

- triptans have the lowest risk of relapse, ${ }^{44}$
- simple analgesics have a higher risk of relapse than triptans, ${ }^{22,44}$ and

- opioids have the highest risk of relapse. ${ }^{22}$

I Where the data don't agree. Data on combination analgesics and on ergots are conflicting. ${ }^{22}$ In addition, data on whether the primary type of headache predicts relapse rate conflict; however, migraine might predict a better outcome than tension-type headache. ${ }^{22}$

\section{To recap and expand: Management pearls}

The major goals of headache management generally are to rule out secondary headache, reach a correct diagnosis, reduce overall headache frequency, and provide effective abortive medication. A large component of reducing headache frequency is addressing and treating medication overuse.

I Seek to understand the nature of the patient's headache disorder. Components of the history are key in identifying the underlying headache diagnosis and ruling out other, more concerning secondary headache diagnoses. The ICHD-3 is an excellent resource for treating headache disorders because the classification lists specific diagnostic criteria for all recognized headache diagnoses.

Medication withdrawal-with or without preventive medication-should reduce the frequency of $\mathrm{MOH}$ in 2 or 3 months. If headache does not become less frequent, however, the headache diagnosis might need to be reconsidered. Minimizing the use of abortive medication is generally recommended, but reduction or withdrawal of these medications does not guarantee that patients will revert to an episodic pattern of headache.

Treating withdrawal symptoms is a reasonable approach in some patients, but evidence does not support routinely providing bridging therapy.

I Apply preventives carefully. Abortive medication withdrawal should generally be completed before initiating preventive medication; however, over the short term, starting preventive therapy while withdrawing the overused medication could assist in reducing headache frequency rapidly. This strategy 
can put patients at risk of medication adverse effects and using the medications longer than necessary, yet might be reasonable in certain patients, given their comorbidities, risk of relapse, and physician and patient preference. A preventive medication for an individual patient should generally be chosen in line with recommendations of the American Academy of Neurology ${ }^{45}$ and on the basis of the history and comorbidities.

I Provide education, which is essential to lowering barriers to success. Patients with $\mathrm{MOH}$ must be counseled to understand that (1) a headache treatment that is supposed to be making them feel better is, in fact, making them feel worse and (2) they will get worse before they get better. Many patients are afraid to be without medication to use as needed. It is helpful to educate them on the different types of treatments (abortive, preventive); how $\mathrm{MOH}$ interferes with headache prophylaxis and medication efficacy; how $\mathrm{MOH}$ alters brain function (ie, aforementioned physiologic changes in pain processing and functional imaging changes ${ }^{23}$ ); and that such change is reversible when medication is withdrawn.

ACKNOWLEDGEMENT

The author thanks Jeffrey Curtis, MD, MPH, for his support and editing assistance with the manuscript.

CORRESPONDENCE

Allison Crain, MD, 2927 N 7th Avenue, Phoenix, AZ 85013; Allison.Crain@dignityhealth.org.

References

1. Zeeberg P, Olesen J, Jensen R. Discontinuation of medication overuse in headache patients: recovery of therapeutic responsiveness. Cephalalgia. 2006;26:1192-1198.

2. Kristoffersen ES, Straand J, Vetvik KG, et al. Brief intervention for medication-overuse headache in primary care. The BIMOH study: a double-blind pragmatic cluster randomised parallel controlled trial. J Neurol Neurosurg Psychiatry. 2015;86:505-512.

3. Bahra A, Walsh M, Menon S, et al. Does chronic daily headache arise de novo in association with regular use of analgesics? Headache. 2003;43:179-190.

4. Blumenfeld AM, Varon SF, Wilcox TK, et al. Disability, HRQoL and resource use among chronic and episodic migraineurs: results from the International Burden of Migraine Study (IBMS) Cephalalgia. 2011;31:301-315.

5. Chu H-T, Liang C-S, Lee J-T, et al. Associations between depression/anxiety and headache frequency in migraineurs: a crosssectional study. Headache. 2018;58:407-415.

6. Bigal ME, Lipton RB. Excessive acute migraine medication use and migraine progression. Neurology. 2008;71:1821-1828.

7. Colás R, Muñoz P, Temprano R, et al. Chronic daily headache with analgesic overuse: epidemiology and impact on quality of life. Neurology. 2004;62:1338-1342.

8. Linde M, Gustavsson A, Stovner LJ, et al. The cost of headache disorders in Europe: the Eurolight project. Eur J Neurol. 2012;19: 703-711.
9. Shah AM, Bendtsen L, Zeeberg P, et al. Reduction of medication costs after detoxification for medication-overuse headache. Headache. 2013;53:665-672.

10. GBD 2016 Headache Collaborators. Global, regional, and nation al burden of migraine and tension-type headache, 1990-2016: a systematic analysis for the Global Burden of Disease Study 2016 Lancet Neurol. 2018;17:954-976.

11. Kernick D, Stapley S, Goadsby PJ, et al. What happens to new-onse headache presenting to primary care? A case-cohort study using electronic primary care records. Cephalalgia. 2008;28:1188-1195.

12. Stone J, Carson A, Duncan R, et al. Who is referred to neurology clinics? - the diagnoses made in 3781 new patients. Clin Neuro Neurosurg. 2010;112:747-751.

13. Munoz-Ceron J, Marin-Careaga V, Peña L, et al. Headache at the emergency room: etiologies, diagnostic usefulness of the ICHD 3 criteria, red and green flags. PloS One. 2019;14:e0208728.

14. Evers S, Marziniak M. Clinical features, pathophysiology, and treatment of medication-overuse headache. Lancet Neurol. 2010;9:391-401.

15. Tassorelli $\mathrm{C}$, Jensen $\mathrm{R}$, Allena $\mathrm{M}$, et al; the COMOESTAS Consortium. A consensus protocol for the management of medicationoveruse headache: evaluation in a multicentric, multinational study. Cephalalgia. 2014;34:645-655.

16. Bigal ME, Serrano D, Buse D, et al. Acute migraine medications and evolution from episodic to chronic migraine: a longitudinal population-based study. Headache. 2008;48:1157-1168.

17. Headache Classification Committee of the International Headache Society (IHS). The International Classification of Headache Disorders, 3rd edition. Cephalalgia. 2018;38:1-211.

18. Ferrari A, Leone S, Vergoni AV, et al. Similarities and differences between chronic migraine and episodic migraine. Headache. 2007;47:65-72.

19. Hagen K, Linde M, Steiner TJ, et al. Risk factors for medicationoveruse headache: an 11-year follow-up study. The Nord-Trøndelag Health Studies. Pain. 2012;153:56-61.

20. Katsarava Z, Schneewiess S, Kurth T, et al. Incidence and predictors for chronicity of headache in patients with episodic migraine. Neurology. 2004;62:788-790.

21. Lipton RB, Fanning KM, Buse DC, et al. Migraine progression in subgroups of migraine based on comorbidities: results of the CaMEO study. Neurology. 2019;93:e2224-e2236.

22. Munksgaard SB, Madsen SK, Wienecke T. Treatment of medication overuse headache-a review. Acta Neurol Scand. 2019;139:405-414.

23. Ferraro S, Grazzi L, Mandelli M, et al. Pain processing in medication overuse headache: a functional magnetic resonance imaging (fMRI) study. Pain Med. 2012;13:255-262.

24. Diener H-C, Holle D, Solbach K, et al. Medication-overuse headache: risk factors, pathophysiology and management. Nat Rev Neurol. 2016;12:575-583.

25. Limmroth V, Katsarava Z, Fritsche G, et al. Features of medication overuse headache following overuse of different acute headache drugs. Neurology. 2002;59:1011-1014.

26. Mauskop A, ed. Migraine and Headache. 2nd ed. Oxford University Press; 2013.

27. Diener H-C, Bussone G, Van Oene IC, et al; TOPMAT-MIG201(TOP-CHROME) Study Group. Topiramate reduces headache days in chronic migraine: a randomized, double-blind, placebocontrolled study. Cephalalgia. 2007;27:814-823.

28. Navratilova E, Behravesh S, Oyarzo J, et al. Ubrogepant does not induce latent sensitization in a preclinical model of medication overuse headache Cephalalgia. 2020;40:892-902.

29. Kristoffersen ES, Straand J, Russell MB, et al. Lasting improvement of medication-overuse headache after brief interventiona long-term follow-up in primary care. Eur J Neurol. 2017;24 883-891.

30. Carlsen LN, Munksgaard SB, Jensen RH, et al. Complete detoxification is the most effective treatment of medication-overuse headache: a randomized controlled open-label trial. Cephalalgia. 2018;38:225-236.

31. Sarchielli P, Messina P, Cupini LM, et al; SAMOHA Study Group. Sodium valproate in migraine without aura and medication overuse headache: a randomized controlled trial. Eur Neuropsychopharmacol. 2014;24:1289-1297.

32. Hagen K, Stovner LJ. A randomized controlled trial on medication-overuse headache: outcome after 1 and 4 years. Acta Neurol Scand Suppl. 2011;124(suppl 191):38-43.

33. Munksgaard SB, Bendtsen L, Jensen RH. Detoxification of
Inpatient care of withdrawal might be beneficial when a patient has a severe comorbidity; does not respond to, tolerate, or adhere to treatment; or relapses after withdrawal. 
medication-overuse headache by a multidisciplinary treatment programme is highly effective: a comparison of two consecutive treatment methods in an open-label design. Cephalalgia. 2012;32:834-844.

34. Silberstein S, Lipton R, Dodick D, et al. Topiramate treatment of chronic migraine: a randomized, placebo-controlled trial of quality of life and other efficacy measures. Headache. 2009;49:1153-1162.

35. Silberstein SD, Blumenfeld AM, Cady RK, et al. OnabotulinumtoxinA for treatment of chronic migraine: PREEMPT 24-week pooled subgroup analysis of patients who had acute headache medication overuse at baseline. J Neurol Sci. 2013;331:48-56.

36. Sandrini G, Perrotta A, Tassorelli C, et al. Botulinum toxin type-A in the prophylactic treatment of medication-overuse headache: a multicenter, double-blind, randomized, placebo-controlled, parallel group study. J Headache Pain. 2011;12:427-433.

37. Tepper SJ. CGRP and headache: a brief review. Neurol Sci. 2019;40(suppl 1):99-105

38. Diener H-C, Dodick D, Evers S, et al. Pathophysiology, prevention and treatment of medication overuse headache. Lancet Neurol. 2019;18:891-902

39. Krymchantowski AV, Barbosa JS. Prednisone as initial treatment of analgesic-induced daily headache. Cephalalgia. 2000;20: 107-113.
40. Bøe MG, Mygland A, Salvesen R. Prednisolone does not reduce withdrawal headache: a randomized, double-blind study. Neurology. 2007;69:26-31.

41. Paolucci M, Altamura C, Brunelli N, et al. Methylprednisolone plus diazepam iv as bridge therapy for medication overuse headache. Neurol Sci. 2017;38:2025-2029.

42. Taghdiri F, Togha M, Razeghi Jahromi S, et al. Celecoxib vs prednisone for the treatment of withdrawal headache in patients with medication overuse headache: a randomized, double-blind clinical trial. Headache. 2015;55:128-135.

43. Ramsey RR, Ryan JL, Hershey AD, et al. Treatment adherence in patients with headache: a systematic review. Headache. 2014;54:795-816.

44. Katsarava Z, Muessig M, Dzagnidze A, et al. Medication overuse headache: rates and predictors for relapse in a 4-year prospective study. Cephalalgia. 2005;25:12-15.

45. Silberstein SD, Holland S, Freitag F, et al; Quality Standards Subcommittee of the American Academy of Neurology and the American Headache Society. Evidence-based guideline update: pharmacologic treatment for episodic migraine prevention in adults: report of the Quality Standards Subcommittee of the American Academy of Neurology and the American Headache Society. Neurology. 2012; 78:1137-1145.

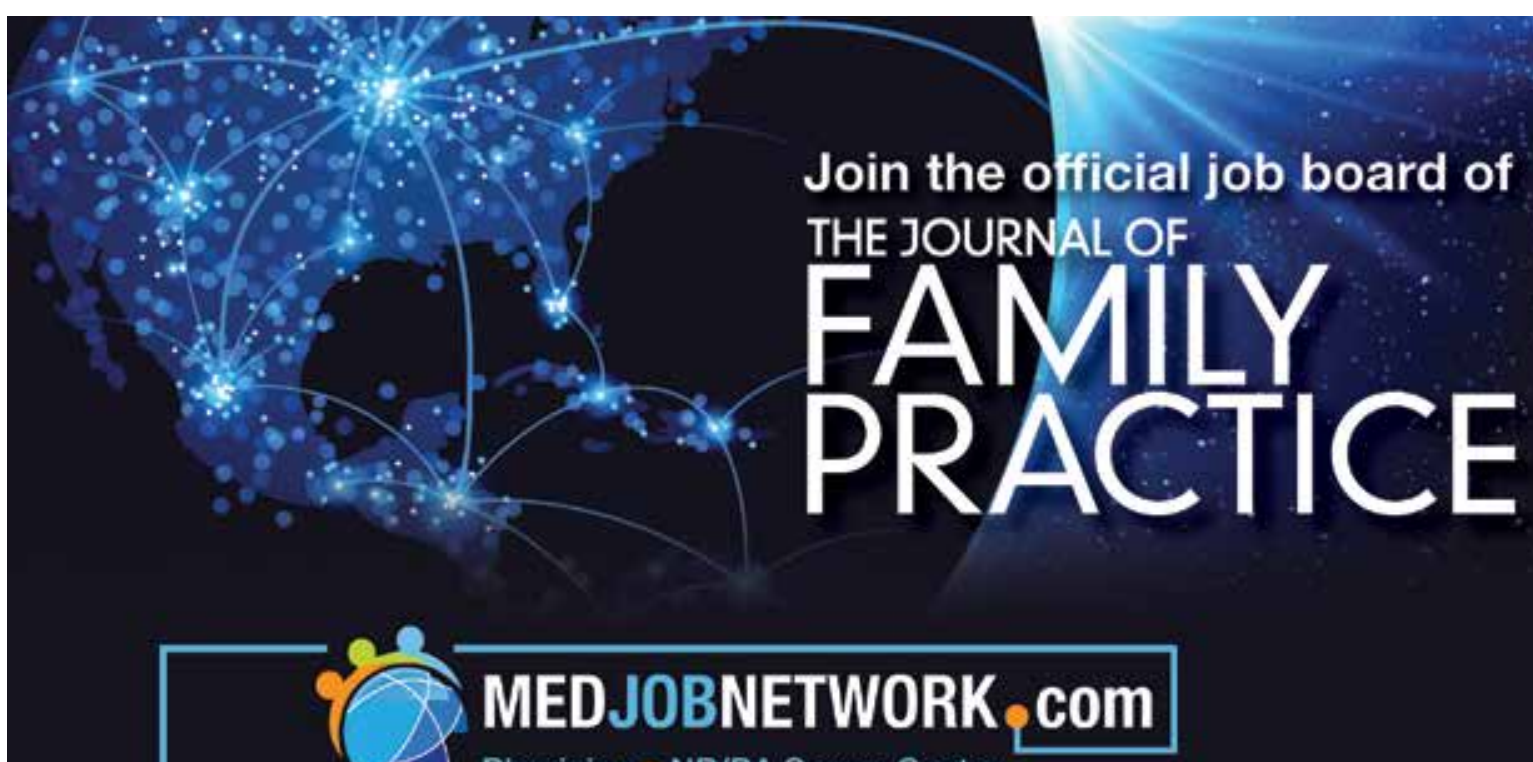

Physician $\bullet$ NP/PA Career Center

Search 1000 s of Jobs and Apply in 1 Click And get FREE benefits including...

- Access to $30+$ medical web sites

- Medical news and clinical advice

- E-Alert and Newsletters on your smart phone

- Online CME and MD-IQ Quizzes

- Coverage of over 200 meetings

And much more!

Julian Knight

Digital Sales Director, Classifieds Phone: 973-206-2317

jknight@frontlinemedcom.com www.medjobnetwork.com 